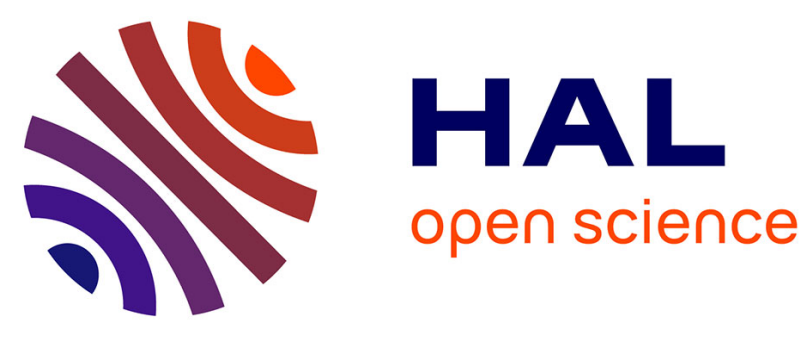

\title{
Meules antiques de Reims
}

Florent Jodry, Philippe Rollet, Paul Picavet, François Moiret, Gilles Fronteau, Agnès Balmelle

\section{- To cite this version:}

Florent Jodry, Philippe Rollet, Paul Picavet, François Moiret, Gilles Fronteau, et al.. Meules antiques de Reims. Olivier Buchsenschutz; Stéphanie Lepareux-Couturier; Gilles Fronteau. Les Meules du Néolithique à l'époque médiévale: technique, culture, diffusion. Actes du 2ème colloque du Groupe Meule, Reims, du 15 au 17 mai 2014, 43, Société archéologique de l'Est; ARTEHIS Éditions, pp.135145, 2017, Revue archéologique de l'Est, supplément, 2-915544-37-9. 10.4000/books.artehis.3286 . hal-02957053

\section{HAL Id: hal-02957053 https://hal.science/hal-02957053}

Submitted on 4 Oct 2020

HAL is a multi-disciplinary open access archive for the deposit and dissemination of scientific research documents, whether they are published or not. The documents may come from teaching and research institutions in France or abroad, or from public or private research centers.
L'archive ouverte pluridisciplinaire HAL, est destinée au dépôt et à la diffusion de documents scientifiques de niveau recherche, publiés ou non, émanant des établissements d'enseignement et de recherche français ou étrangers, des laboratoires publics ou privés. 
Revue Archeologique de l'Est

\section{Les meules du Néolithique à Yépoque médlévale :} technique, culturie, diffusion

Actes du $2^{\text {ème }}$ colloque du Groupe Meule, Reims, du 15 au 17 mai 2014
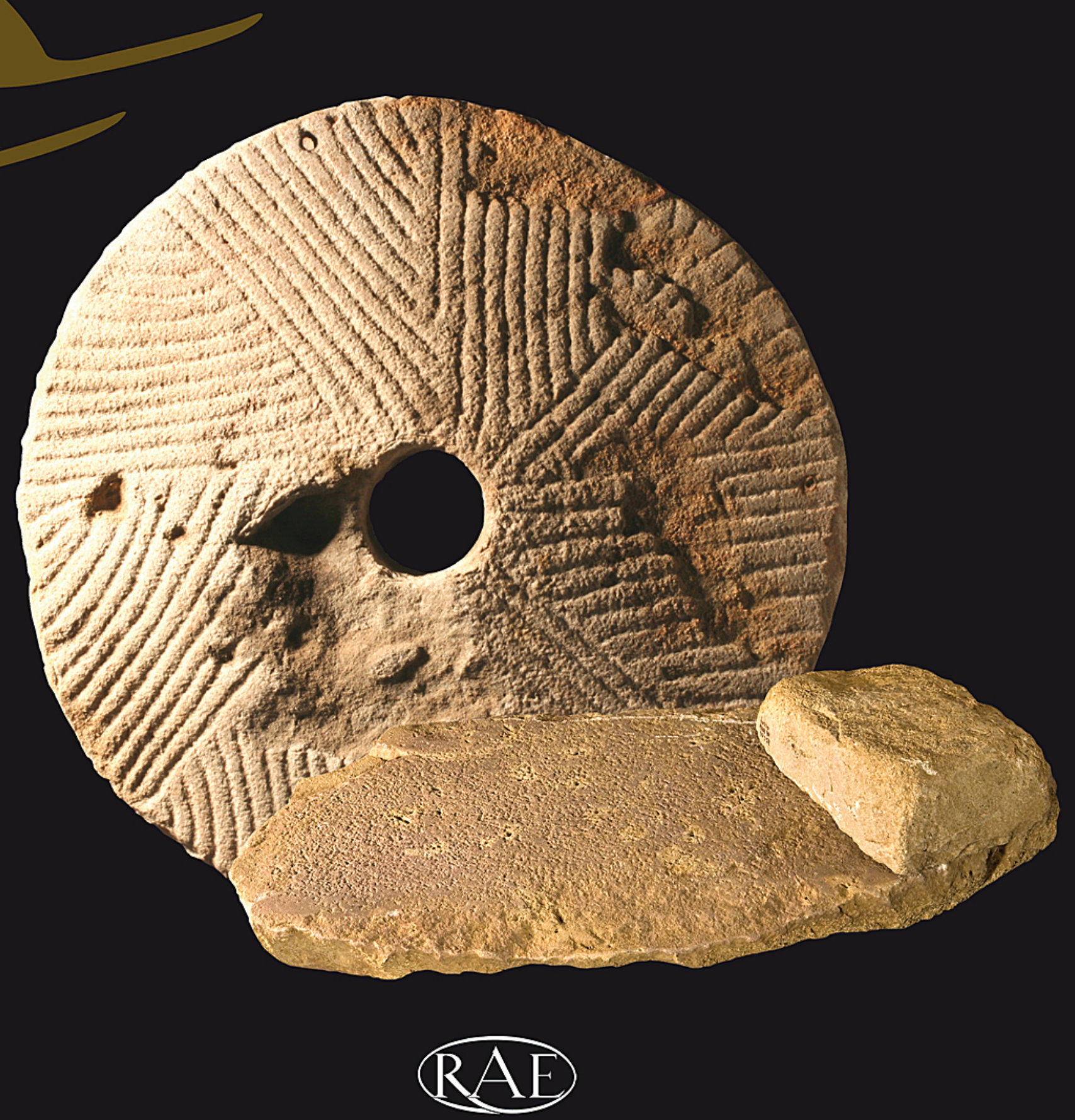

Dijon 2017 


\title{
MEULES ANTIQUES DE REIMS
}

\author{
F. Jodry, P. Rollet, P. Picavet, Fr. Moiret et G. Fronteau, \\ avec la coll. d'A. BALMELLE
}

\begin{abstract}
Mots-clés Reims, meules, échanges commerciaux, voies de communication, transports.
Keywords Reims, rotary querns, millstones, trade, roads, transport, Antiquity.
\end{abstract}

Résumé Plus de 70 meules ont été découvertes dans la ville de Reims ces dernières années auxquelles s'ajoutent d'anciennes découvertes réalisées en grande majorité dans le centre historique de la ville. De grands corpus comme ceux de la rue Maucroix ou du Boulevard Henrot nous permettent d'effectuer de nouvelles observations morphologiques et pétrographiques, à la lumière des nouvelles avancées dans la thématique des moulins rotatifs.

L'analyse que nous proposons ici repose sur la cartographie de l'ensemble des découvertes anciennes et récentes dans la ville antique en corrélation avec les nombreuses voies de communication qui la desservent. Reliée aux principales villes du nord de la Gaule et aux provinces du sud par la voie Agrippa, la capitale de la Gaule Belgique constitue une étape importante dans le transport et les échanges commerciaux.

\begin{abstract}
More than 70 querns and millstones have been brought to light in recent years in the city of Reims, France. A series of old finds, primarily from the city's centre, can be added to the lot. The study of the large assemblages, such as those of the excavated settlements of Rue Maucroix or Boulevard Henrot, enable us to propose new morphological and petrographical observations in the framework of recent advances on the subject of rotary mills.

The analysis presented in this paper is based on a map correlating the spread of the city's querns and millstones with the many ancient trade routes. The capital of Gallia Belgica (Durocortorum) played an important role in the transport and trade of millstones between the main centres in northern Gaul and the southern provinces by means of the Via Agrippa.
\end{abstract}

\section{LE SITE DE REIMS}

Entre la fin de la période gauloise et le milieu du $\mathrm{IV}^{\mathrm{e}} \mathrm{s}$. ap. J.-C., trois enceintes se succèdent sur le site de Reims (fig. 1). La plus ancienne, datée de la fin de la période gauloise est de peu postérieure aux années 70-60 av. J.-C. (BALMELLE, 1995) et correspond à un fossé large de $45 \mathrm{~m}$ à l'ouverture, profond de 5 à $10 \mathrm{~m}$ qui enclot une surface de 90 ha (BERTHELOT et alii, 1993 ; NEISS, ROLLET, 2011). Au cours de la première partie du règne d'Auguste, Durocortorum, capitale de la Gaule Belgique, se dote d'une nouvelle enceinte: un fossé de plus de $7 \mathrm{~km}$ de longueur, précédé d'une levée de terre, s'appuie au nord et au sud sur la rivière (Vesle) et porte l'extension urbaine à près de 600 ha (NeIss et alii, 2007). Enfin, le mur de l'Antiquité tardive construit au $\mathrm{IV}^{\mathrm{e}} \mathrm{s}$. reprend les quatre arcs monumentaux édifiés vers la fin du $\mathrm{II}^{\mathrm{e}} \mathrm{s}$.-début du $\mathrm{III}^{\mathrm{e}} \mathrm{s}$., réduisant la surface enclose à 55 ha (NEISS, SINDONINO, 2004). Ces trois enceintes sont concentriques, le centre étant matérialisé par le croisement des axes majeurs cardo et decumanus qui subsistent dans la topographie urbaine actuelle. Au sein de ce vaste espace, de nombreuses opérations de fouille préventives ont eu lieu et plusieurs thèmes peuvent être abordés grâce à une documentation de plus en plus fournie.

L'étude menée par le PCR a permis de réaliser un premier recensement des éléments de mouture recueillis lors des opérations archéologiques ou provenant de découvertes anciennes. Ce travail est considérable et bien que certaines pièces soient aujourd'hui manquantes, il est nécessaire de prendre en compte le fait qu'à Reims les collections issues des chantiers d'archéologie préventive ont voyagé d'un dépôt municipal à un autre, chaque déménagement ayant occasionné des pertes de renseignements (indications de provenance), voire la perte d'un ou plusieurs objet(s). Finalement sur près de soixante chantiers, seuls quatorze ont livré des éléments liés à la mouture (fig. 2 et fig. 3). La répartition n'est pas homogène sur l'ensemble de la ville antique (fig. 2), et on constate que des chantiers effectués sur des surfaces importantes, sur lesquelles des îlots urbains ont été en grande partie dégagés, n'ont livré aucun élément: 


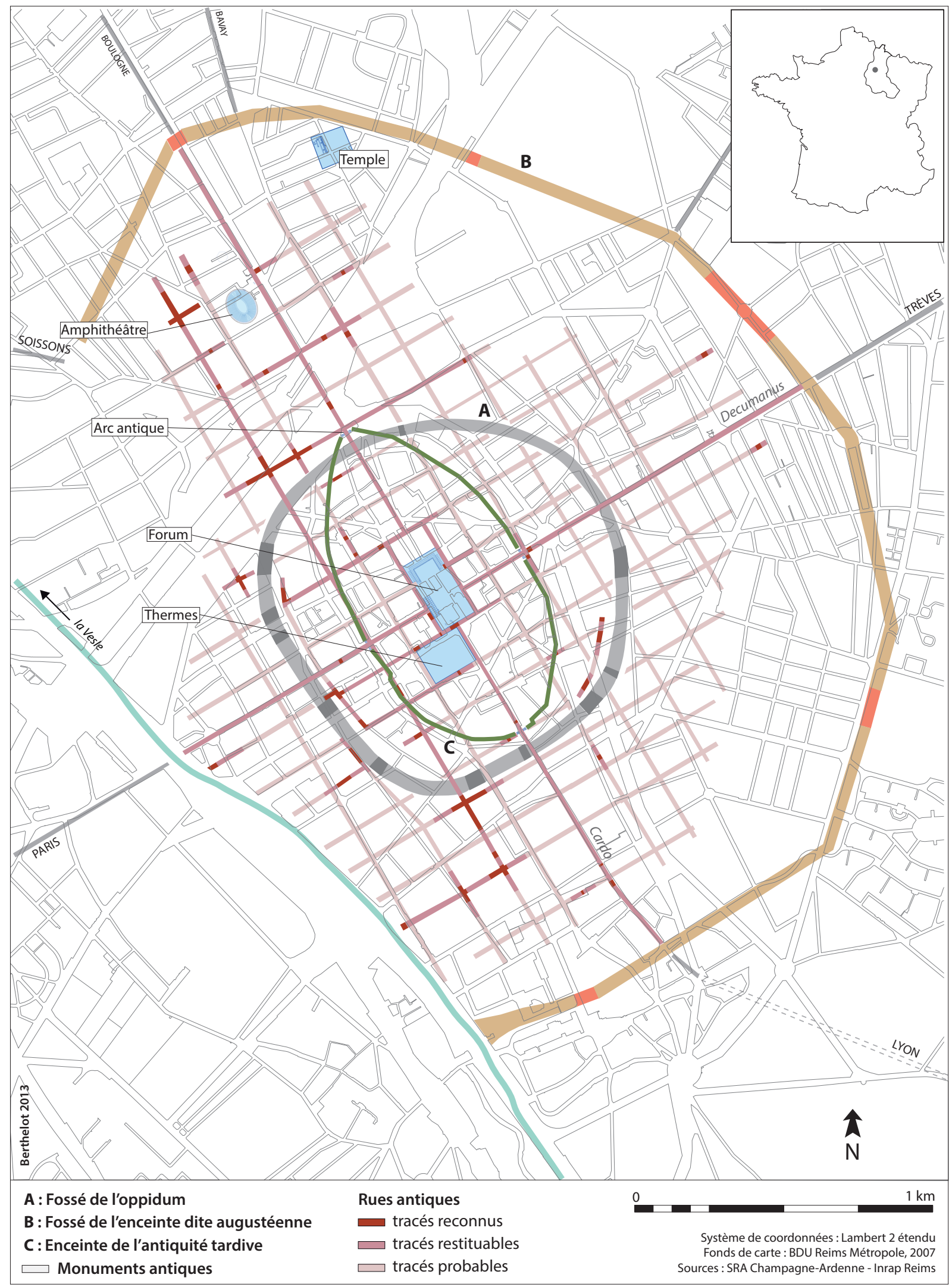

Fig. 1. Plan de la ville actuelle sur lequel est reporté le tracé des différentes enceintes, les rues et les principaux monuments antiques. 


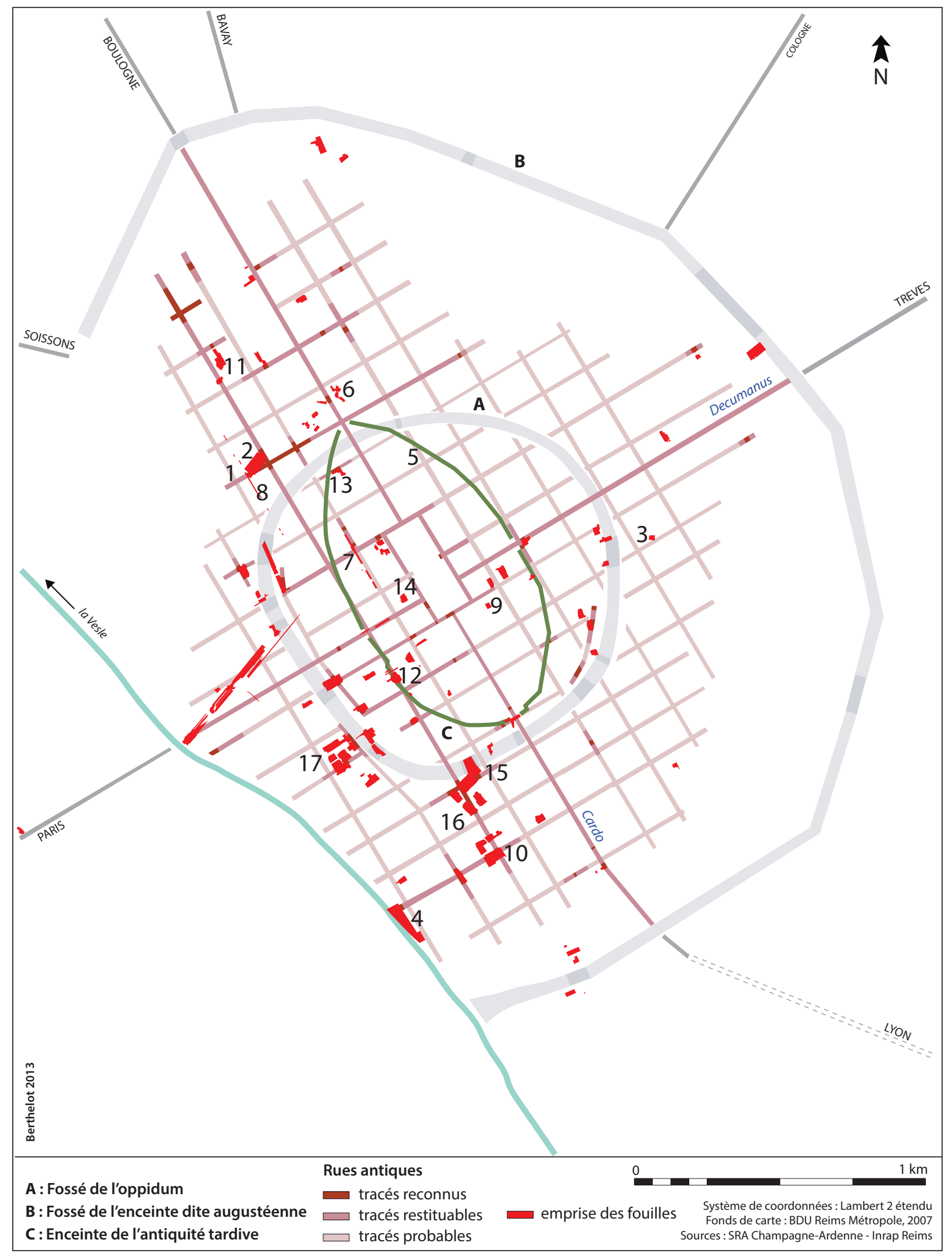

Fig. 2. Localisation des principales opérations archéologiques. 


\begin{tabular}{|c|c|c|c|c|}
\hline & Opérations & Surface & Nb et type & Datation \\
\hline 1 & gare SNCF & (?) & $1(\mathrm{C})$ & GR? \\
\hline 2 & 28, bd Joffre & $4000 \mathrm{~m}^{2}$ & $2(\mathrm{M})$ & $\| I^{e}-I I^{e}$ \\
\hline 3 & 36 bis-38, rue de Cernay & $280 \mathrm{~m}^{2}$ & $1(\mathrm{C})$ & 80-90 à 120 ap. J.-C. \\
\hline 4 & $\begin{array}{l}\text { Bd Henri Henrot } \\
\text { (Zac du Vieux Port) }\end{array}$ & $4200 \mathrm{~m}^{2}$ & $10(5 \mathrm{C}-5 \mathrm{M})$ & début IIIe - début IVe \\
\hline 5 & rue du Temple & $200 \mathrm{~m}^{2}$ & $1(C)$ & GR? \\
\hline 6 & $\begin{array}{l}\text { Place de la République } \\
\text { (Tramway) }\end{array}$ & $155 \mathrm{~m}^{2}$ & $2(C)$ & fin III - début IVe \\
\hline 7 & $\begin{array}{l}\text { Cours J.-B. Langlet } \\
\text { (Tramway) }\end{array}$ & $150 \mathrm{~m}^{2}$ & $1(\mathrm{C})$ & GR? \\
\hline 8 & $\begin{array}{l}\text { Bd L. Roederer } \\
\text { (Tramway) }\end{array}$ & $6500 \mathrm{~m}^{2}$ & $7(6 C-1 M)$ & début Ier-fin III \\
\hline 9 & rue St-Symphorien & $300 \mathrm{~m}^{2}$ & $1(\mathrm{M})$ & fin III'- début IVe \\
\hline 10 & rue de l'Équerre & $7500 \mathrm{~m}^{2}$ & $3(1 C-2 M)$ & $\begin{array}{l}5 \text { av. J.- C à } 20 \text { ap. J.-C. } \\
\text { et } 40 \text { à } 70 \text { ap. J.-C. }\end{array}$ \\
\hline 11 & $\begin{array}{l}\text { rues Maucroix- } \\
M^{t} d^{\prime} \text { 'Arène }\end{array}$ & $3000 \mathrm{~m}^{2}$ & $13(7 \mathrm{C}-6 \mathrm{M})$ & fin ler $^{\text {- fin }}$ III $^{e}$ \\
\hline 12 & $\begin{array}{l}\text { rue Rockefeller } \\
\text { (Médiath. Cathédrale) }\end{array}$ & $1700 \mathrm{~m}^{2}$ & $7(4 C-3 M)$ & 20 à 5 av. J.-C. et II IV \\
\hline 13 & rue du G' Sarrail & $650 \mathrm{~m}^{2}$ & $1(?)$ & fin $\mathrm{l}^{\mathrm{er}}-\mathrm{fin} \mathrm{III}^{\mathrm{e}}$ \\
\hline 14 & rue Carnot & $1000 \mathrm{~m}^{2}$ & $5(3 C-2 M)$ & fin III \\
\hline
\end{tabular}

Fig. 3. Tableau récapitulatif des opérations ayant livré des éléments de mouture ( $M=$ meta, meule dormante / $C=$ catillus, meule tournante). par exemple les opérations mitoyennes des rues Gambetta (BERTHELOT et alii, 1993) et Venise (ROLLET et alii, 2001) qui couvrent à elles seules 1,5 ha (fig. $2, \mathrm{n}^{\text {os }} 15$ et 16) ou encore l'îlot Capucins-Hincmar-Clovis (BALMELLE et alii, 1990), d'une surface de $7500 \mathrm{~m}^{2}$ (fig. 2, n 17). Deux opérations ont cependant livré des lots importants : rue Maucroix dans la partie nord de la ville (RoLLET et alii, 2011) (fig. 2, $\left.\mathrm{n}^{\circ} 11\right)$ avec quatorze pièces et bd Henri Henrot avec dix pièces (ROLLET, 2016). Celui-ci se trouve dans la partie sud de la ville, en bordure de la rivière et à proximité du quartier artisanal des ateliers de potiers (fig. 2, $n^{\circ} 4$ ).

En ce qui concerne les datations, la grande majorité des objets a été recueillie dans des contextes chronologiques situés entre le début du $\mathrm{III}^{\mathrm{e}} \mathrm{s}$. et le milieu du IV ${ }^{\mathrm{e}} \mathrm{s}$. Deux opérations ont toutefois livré des éléments plus anciens, datés des deux dernières décennies du $\mathrm{I}^{\mathrm{er}} \mathrm{s}$. av. J.-C. pour la rue Rockefeller (fig. 2, $\mathrm{n}^{\circ}$ 12) et de contextes du $\mathrm{I}^{\mathrm{er}}$ s. ap. J.-C. rue de l'Équerre (fig. 2, $\mathrm{n}^{\circ}$ 10). Cependant, il faut considérer que certaines datations avancées dans les études de chantiers anciens doivent être affinées.

\section{LOCALISATION DES AIRES DE TRAVAIL IDENTIFIÉES OU POTENTIELLES}

À l'exception du chantier de la rue Rockefeller où une aire de battage du blé à été mise au jour, aucune pièce de mouture ne peut être associée avec certitude à un lieu où se déroulait cette activité. L'aire de battage a été dégagée sur une surface de près de $30 \mathrm{~m}^{2}$ (fig. 4) et correspond à

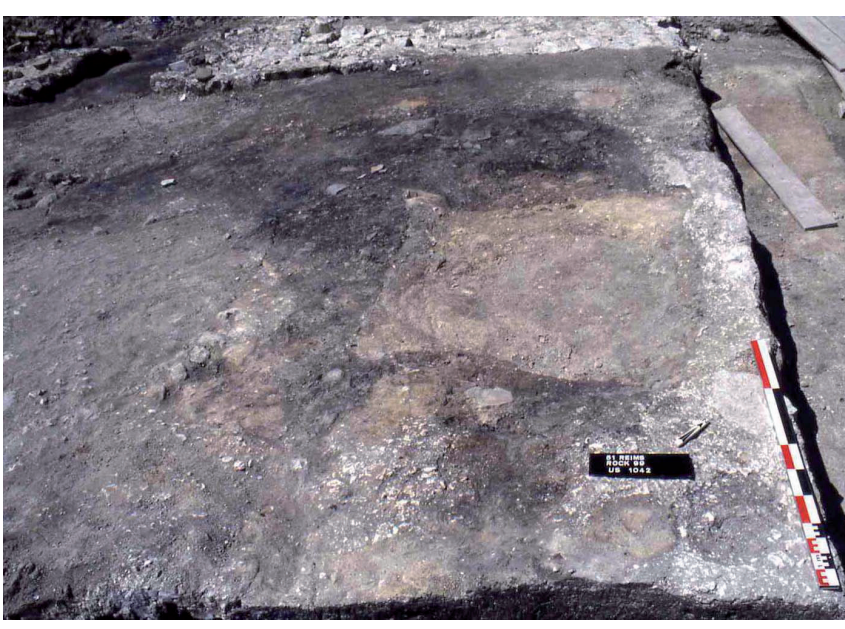

Fig. 4. Vue d'ensemble de l'aire de battage mise au jour sur le site de la rue Rockefeller, Médiathèque centrale; les fragments de meule sont visibles. En noir, grains de blé ou de froments carbonisés (cl. A. Balmelle).

un sol en craie aménagé à même le paléosol (BALMELLE, Sindonino, 2004). Ce sol était tapissé de graines carbonisées (blé tendre) accompagnées de plusieurs fragments de meules également brûlés ont été découverts. L'étude du mobilier céramique permet de situer cette activité au cours de la première partie du règne d'Auguste.

Tout au plus, sur l'ensemble des découvertes peut-on constater que ces éléments se trouvent parfois en remploi, dans des aménagements en pierres maçonnées notamment rue Maucroix (fig. $2, \mathrm{n}^{\circ} 11$ ) où une meule brisée a été uti- 


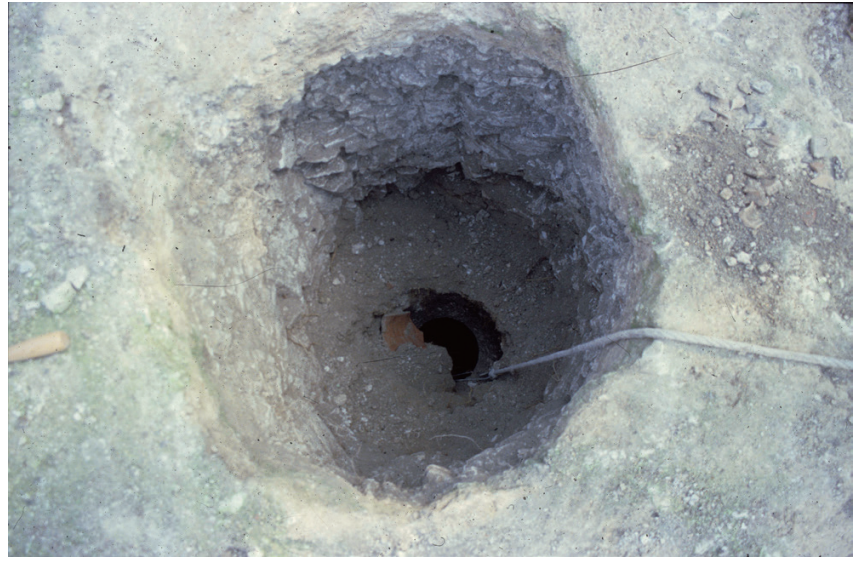

Fig. 5a. Vue zénithale de l'ouverture obstruée du puits de la rue de Cernay (cl. Ph. Rollet).

lisée pour renforcer le sol d'une cour ainsi qu'une autre, entière, qui a servi à garnir le fond d'un caniveau. Mais la grande majorité de ces meules est découverte en contexte de rejet, essentiellement sous forme de fragments, rarement entières. Sur le chantier du tramway un catillus a été recueilli dans le comblement supérieur d'un petit cellier (Sindonino, étude en cours) et rue de Cernay (fig. $2, \mathrm{n}^{\circ} 3$ ), une meule pompéienne entière en basalte obstruait complètement le niveau supérieur d'un puits, préservant un vide de près de $5 \mathrm{~m}$ (ROLLET, 2001 (fig. $5 \mathrm{a}$ et $\mathrm{b}$ ). Quoiqu'il en soit, ces objets retrouvés dans des contextes de remploi ou de rejet sont les témoins d'une activité qui se déroulait très probablement à proximité immédiate.

L'intérêt de cette étude permet également de dresser un bilan à propos de la provenance des éléments de mouture au sein des réseaux commerciaux. En effet, le sous-sol rémois est constitué de craie et bien que les constructeurs gallo-romains aient exploité cette ressource sous différentes formes (pilée et compactée pour la réalisation de sols et de fondations de murs ou en petits moellons pour les murs de cave), ce matériau ne peut en aucun cas être utilisé pour la fabrication de meules.

\section{LES ROCHES}

Les roches des meules observées à Reims sont de quatre sortes, dont trois sédimentaires et une magmatique: les calcaires, la meulière, les grès et le «basalte $»^{1}$.

Les calcaires sont d'abord issus de plusieurs niveaux du Lutétien (Tertiaire) et contiennent différents éléments fossiles caractéristiques. On distingue les calcaires à empreintes de cérithes (Lutétien supérieur), qui constituent sept meules

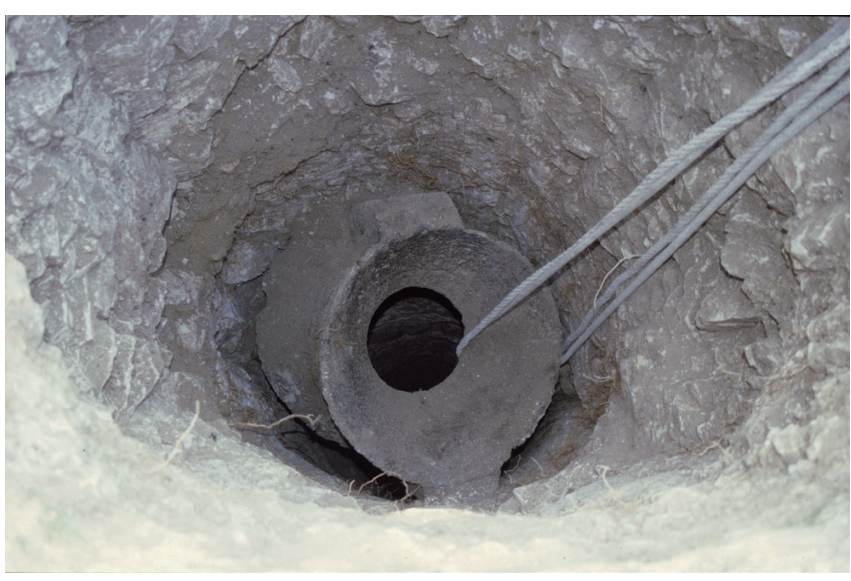

Fig. 5b. Meule pompéienne rejetée dans un puits sur le chantier de la rue de Cernay (cl. Ph. Rollet).

rotatives, les calcaires à ditrupa (Lutétien moyen) pour quatre meules, et les calcaires gréseux à glauconie et rares nummulites (Lutétien inférieur) qui fournissent dix-neuf meules. Les calcaires exploitables les plus proches affleurent à quelques kilomètres à peine de la ville de Reims, sur les versants des vallées qui entaillent les plateaux tertiaires du Bassin parisien. Les niveaux actuellement connus pour avoir réellement fourni des meules rotatives sont plutôt situés dans le Soissonais, trente à cinquante kilomètres à l'ouest de Reims (ROBERT, LANDRÉAT, 2005 ; NAZE et alii, 2011).

La meulière, qui concerne ici un seul individu, est formée au Plio-quaternaire (fin Tertiaire / Quaternaire), est originaire du même bassin sédimentaire que les calcaires (LEPAREUX-COUTURIER et alii, ce vol.).

Deux faciès caractéristiques de grès sont ensuite individualisés. Le grès de type «Fosses-Belleu» (LEPAREUXCOUTURIER et alii, ce vol.), est un grès quartzitique à petits éléments de feldspath blanc et de silex noir (quatre meules), daté de l'Yprésien (Tertiaire). Il est issu de gisements très localisés du nord du Bassin de Paris, et profite probablement des mêmes circuits commerciaux que les calcaires.

Le grès de type «Haybes/Macquenoise» (PICAVET $e t$ alii, ce vol.) est grossier et massif, à tendance feldspathique, et comprend de petits cristaux de tourmaline noire (neuf meules). Il est issu des niveaux dévoniens (Primaire) affleurant sur la bordure occidentale du massif des Ardennes, $90 \mathrm{~km}$ au nord de Reims.

Enfin, le lot «basalte», roche volcanique vacuolaire grise, est représenté par cinq meules. Il provient vraisemblablement des coulées volcaniques quaternaires (Pléistocène) du massif de l'Eifel (Allemagne), à presque $300 \mathrm{~km}$ au nordest de Reims (GLUHAK, HofMEISTER, 2009 et 2011). 


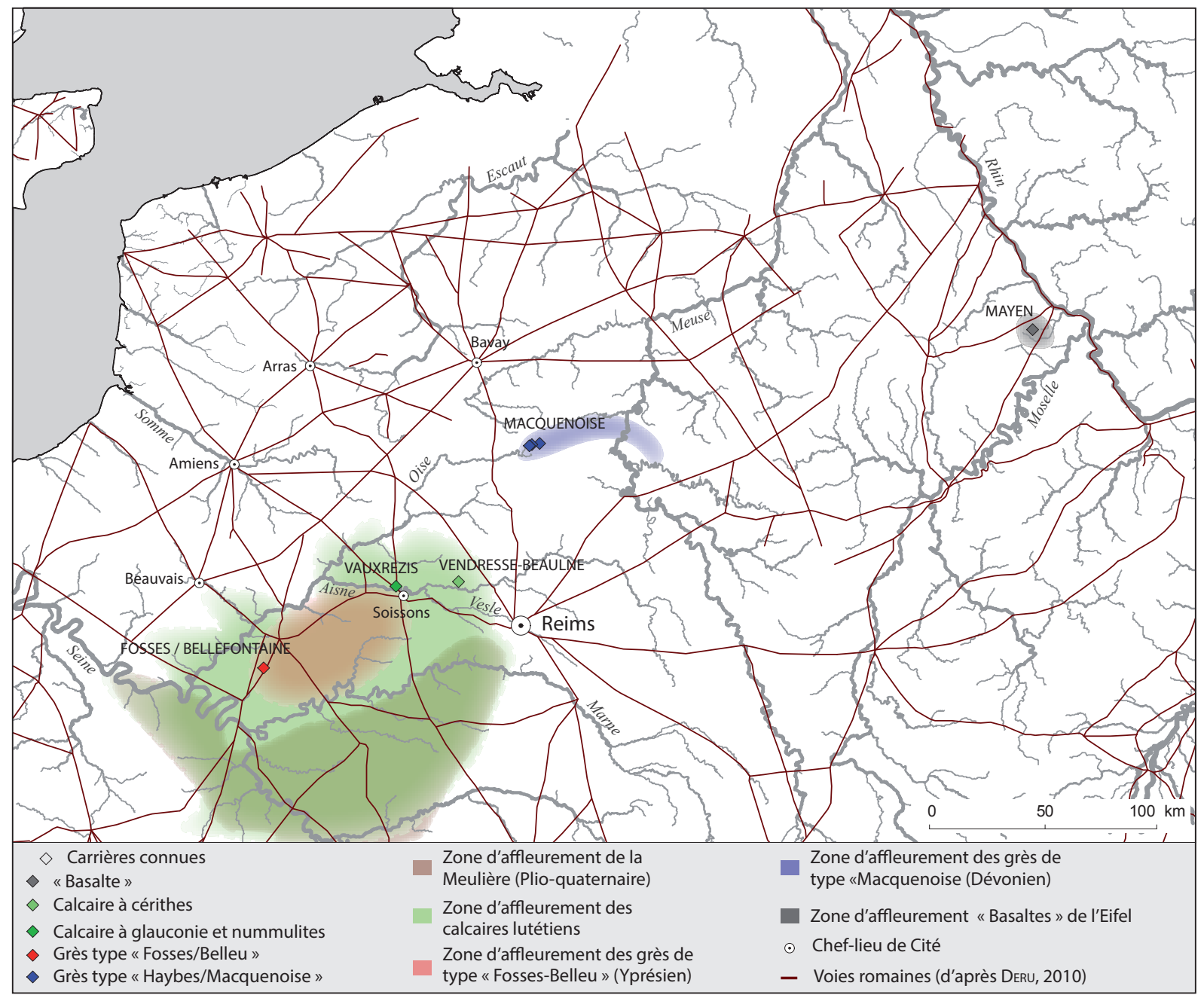

Fig. 6. Voies de communication et origine supposée des meules de Reims à l'époque romaine (carte P. Picavet et $A B G$ ).

\section{RÉSEAUX D’APPROVISIONNEMENT}

L'observation des réseaux de communication organisés autour de la ville de Reims dans l'Antiquité aide à comprendre son approvisionnement en meules (fig. 6). Les productions du Bassin de Paris, en calcaire, meulière et grès de type «Fosses-Belleu » paraissent bénéficier d'un bon réseau, tant fluvial par les vallées de l'Aisne et de la Vesle, que routier par la voie romaine Beauvais - Senlis - Soissons Reims. Il faut pourtant mettre en doute la navigabilité de la Vesle avant sa canalisation au XIX ${ }^{\mathrm{e}}$ s., et envisager un portage terrestre sur $20 \mathrm{~km}$ depuis le cours de l'Aisne au nord, ou de $25 \mathrm{~km}$ depuis celui de la Marne au sud (FrONTEAU et alii, 2014).

En l'absence de voie navigable, les meules des Ardennes et de l'Eifel semblent parvenir à Reims par la route; de plus le secteur carrier de Macquenoise, approvisionnant massivement la ville antique de Bavay (PICAVET et alii, 2011), chef-lieu de la Cité des Nerviens, est situé à une vingtaine de kilomètres de la voie Bavay - Reims.
Les productions du secteur de Mayen (Eifel) remontent le couloir fluvio-routier de la Moselle, et bénéficient des voies mettant en relation Reims et le limes de Germanie.

\section{RÉPARTITION CHRONOLOGIQUE SUR LA VILLE DE REIMS}

La répartition des meules sur la ville de Reims laisse apparaître une nette prédominance des calcaires issus du Bassin de Paris qui constituent $65 \%$ du corpus. Les autres roches d'origine occidentale, grès de type «Fosses-Belleu» et meulière, composent respectivement 7 et $2 \%$ de la série. Les roches originaires du nord-est, grès de type «Haybes/ Macquenoise » et «basaltes» en composent 15 et $9 \%$ (fig. 7a).

Sur les 71 meules prises en compte dans l'étude, trentequatre sont datées au siècle près et permettent d'aborder la chronologie de l'approvisionnement en meules de la ville. L'analyse est cependant biaisée par l'imprécision des datations, puisque ces meules datées ne proviennent que 


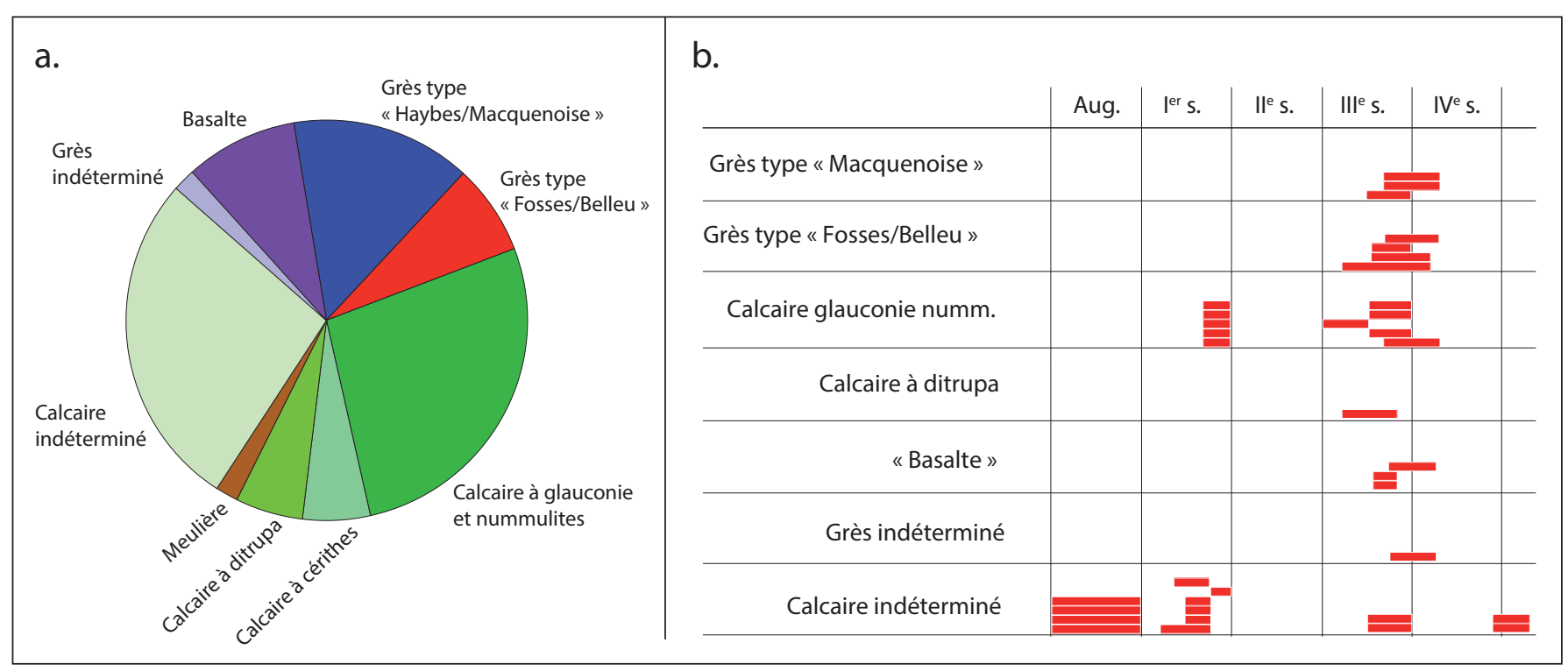

Fig. 7. Proportions et chronologie des roches meulières sur la ville de Reims.

a. Proportions des roches sur toute la série; $\mathbf{b}$. décompte des meules datées au siècle près.

de quatre sites (rue Maucroix, Boulevard Henrot, SaintSymphorien et rue de l'Équerre), les autres étant attribuées à l'Antiquité au sens large.

Il ressort toutefois de cette observation une nette domination des calcaires aux deux premiers siècles de notre ère (fig. 7b). Quand leur composition précise est déterminée, les calcaires identifiés sur la ville de Reims proviennent tous de l'étage du Lutétien du Bassin parisien; il est vraisemblable que les calcaires non reconnus, parce qu'ils ne sont mentionnés que dans d'anciennes publications, en soient issus aussi. L'approvisionnement est alors régional et orienté à l'ouest.

À partir du $\mathrm{III}^{\mathrm{e}}$ siècle, la gamme s'étoffe et se diversifie (fig. 7b); en plus des calcaires, des productions extrarégionales orientales et occidentales sont attestés. De l'Est arrivent les meules du bord occidental du Massifs des Ardennes (grès type «Haybes/Macquenoise») et de l'Eifel («basalte»). De l'Ouest arrivent celles du nord du Bassin de Paris (grès type «Fosses-Belleu»).

Tout au long de l'Antiquité, l'approvisionnement reste donc majoritairement occidental, dans le périmètre régional de la ville accessible par les voies fluviales et routières. $\mathrm{Ce}$ constat correspond au phénomène déjà relevé pour les matériaux de construction des monuments antiques de Reims, dont l'origine est largement située dans les niveaux lutétiens du nord-est du Bassin de Paris (Fronteau et alii, 2014).

L'étude de l'ensemble céramique de la rue Maucroix a par ailleurs montré l'arrivée à Reims de productions extra-régionales d'Argonne et du Noyonnais à partir du milieu du II $^{\mathrm{e}}$ s., et en provenance des Ardennes (Ville-surLumes) à partir du début du $\mathrm{III}^{\mathrm{e}} \mathrm{s}$. seulement (FLORENT, 2007). Précédemment, au début du Haut-Empire, la ville était principalement fournie par les ateliers locaux, et captait ponctuellement les productions du sud de la Gaule en route vers le limes de Germanie.

Un lien semble donc se dessiner entre la hausse au $\mathrm{III}^{\mathrm{e}}$ s. de la part des productions de céramique ardennaise et celle des meules en «arkose» d'Haybes/Macquenoise, pour lesquelles un nouveau courant de diffusion semble se constituer, dirigé cette fois d'est en ouest.

\section{MORPHOLOGIE DES MEULES (fig. 8)}

Les douze collections de meules antiques découvertes sur la ville de Reims, associées aux ensembles du musée Saint-Remi, réunissent 71 meules dont vingt-quatre meules dormantes et quarante-sept meules tournantes. Ce corpus est composé de 62 meules manuelles, quatre meules de type pompéien, quatre meules hydrauliques. Une meule (Re6) est trop mal conservée pour en déterminer le diamètre. La figure 8 présente une sélection des pièces entières.

Les diamètres des meules de cet ensemble se répartissent entre 30 et $95 \mathrm{~cm}$ avec une forte proportion (87\%) de meules au diamètre inférieur ou égal à $55 \mathrm{~cm}$, diamètre délimitant les meules manuelles des meules mécaniques (animale ou hydraulique). Sur ces 62 meules manuelles, cinquante-quatre possèdent un diamètre entre 42 et $44 \mathrm{~cm}$, diamètre «standard» reconnu pour le Haut-Empire (JACCOTTEY et alii, 2011b).

\section{LES AMÉNAGEMENTS}

Les aménagements décrits ici sont de trois types: œils des catillus, manchons d'entraînement pour les catillus manuels et l'habillage des surfaces actives. 


\section{Meules hydrauliques}

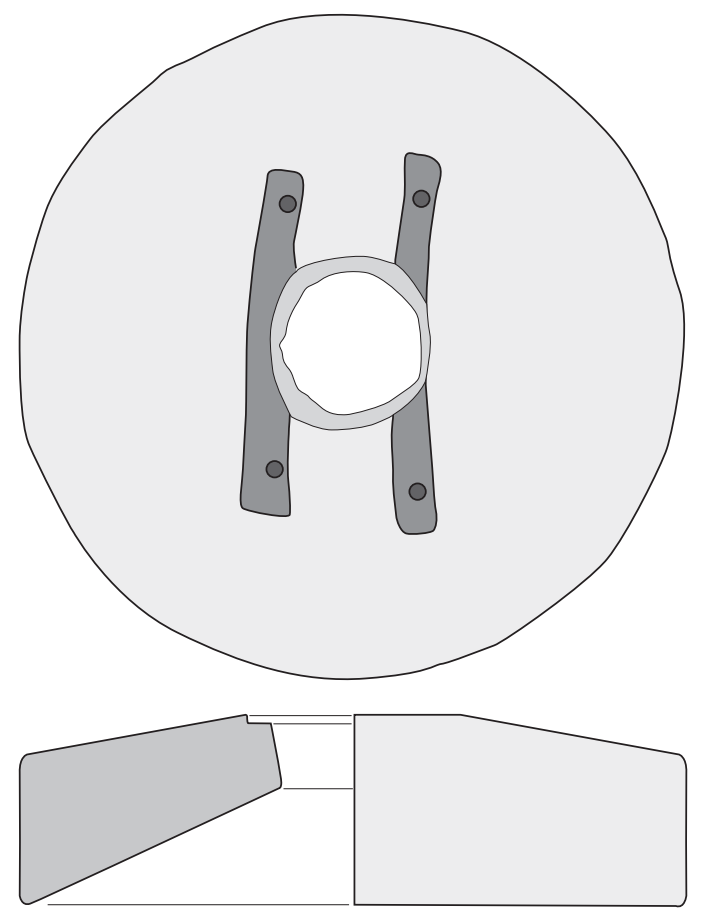

Reims (Marne)

Basalte

Musée Saint-Remi de Reims

Florent Jodry,

Stéphanie Lepareux-Couturier 2009 Étude ${ }^{\circ}$ 51-016 (C132)

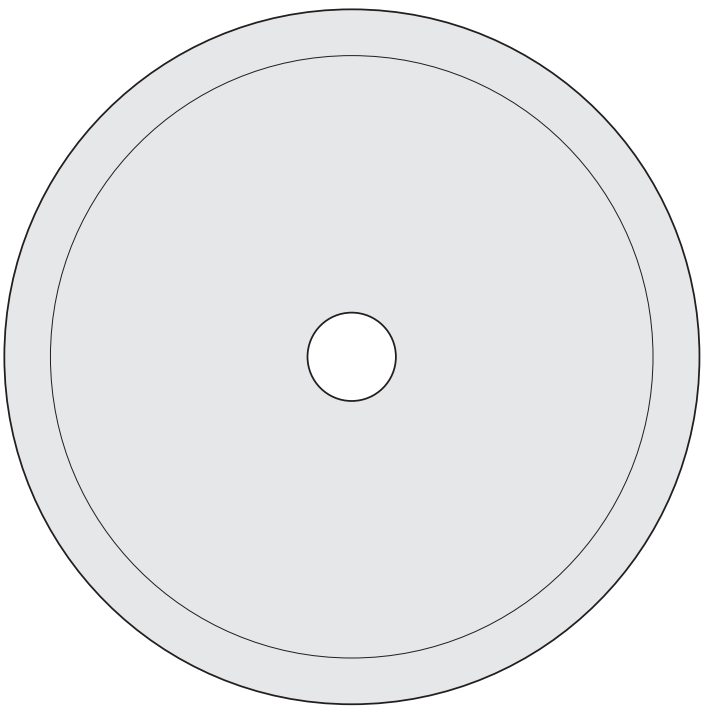

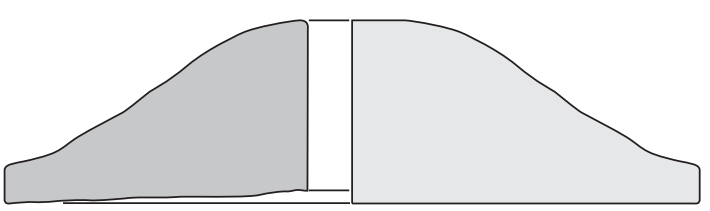

Reims (Marne)

Basalte

Musée Saint-Remi de Reims Florent Jodry,

Stéphanie Lepareux-Couturier 2009 Étude n 51-015 (C146)

\section{Meules manuelles}

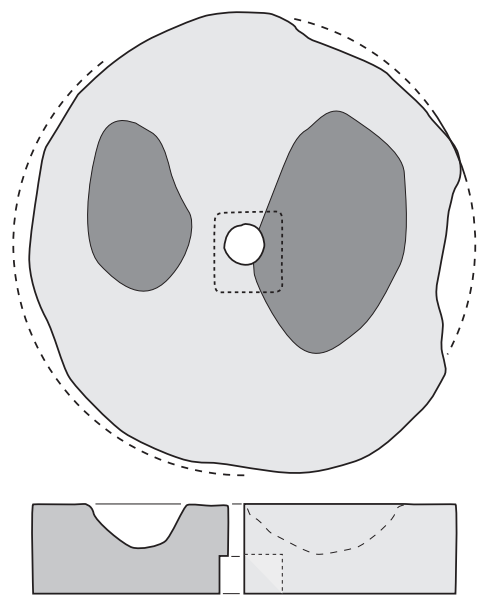

Reims (Marne) - Tram MR349

Calcaire

Centre Inrap Reims

Florent Jodry, 2013

Étude ${ }^{\circ}$ 51-284
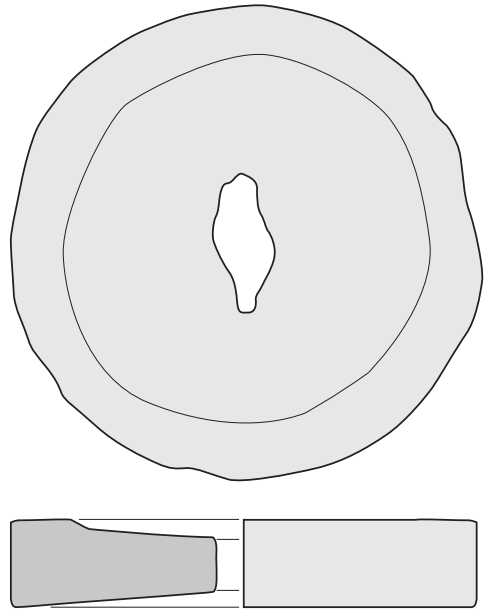

Reims (Marne) - Tram

Fs 24-us7036

Calcaire

Centre Inrap Reims

Florent Jodry, 2013

Étude ${ }^{\circ}{ }^{51-285}$
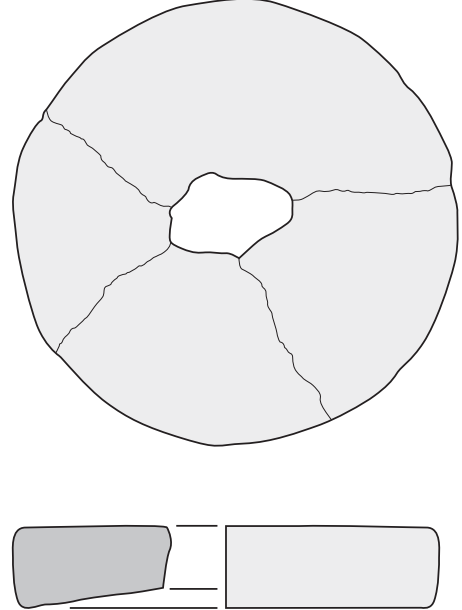

Reims (Marne) - rue Maucroix Ol 686, us 577

Calcaire à glauconie grossière Centre Inrap Reims

Florent Jodry, 2012

Étude ${ }^{\circ}$ 51-035

Fig. 8. Sélection de meules manuelles et hydrauliques de la ville de Reims. 


\section{Meules manuelles}
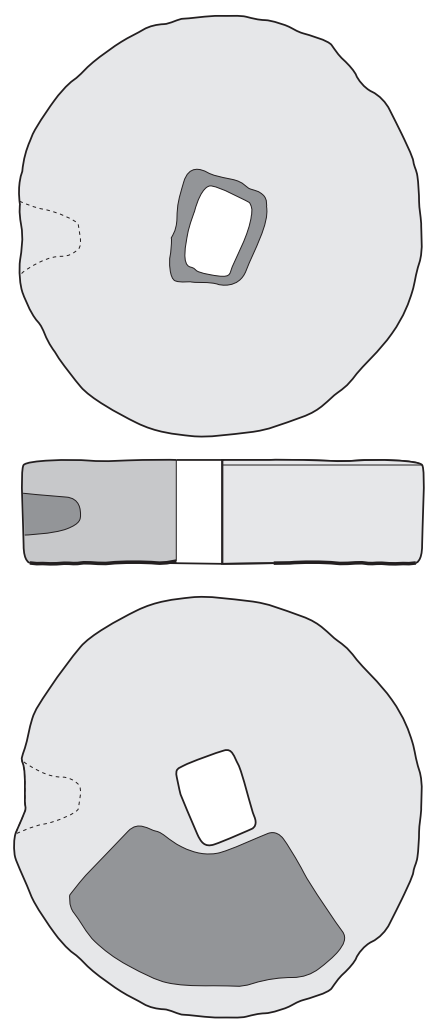

Reims (Marne) - Médiathèque Meulière poreuse Dépôt Mazet/SRA

Florent Jodry, 2013 Étude n 51-298
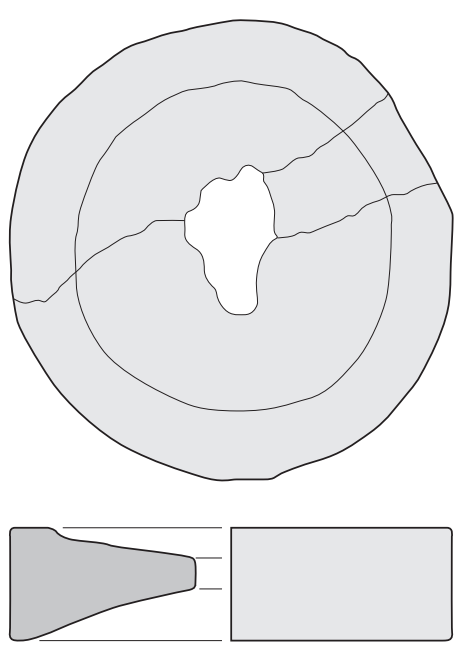

Reims (Marne)

Calcaire coquillier

Reims-Musée Saint-Remi

Florent Jodry, 2014

Étude $n^{\circ}$ 51-309
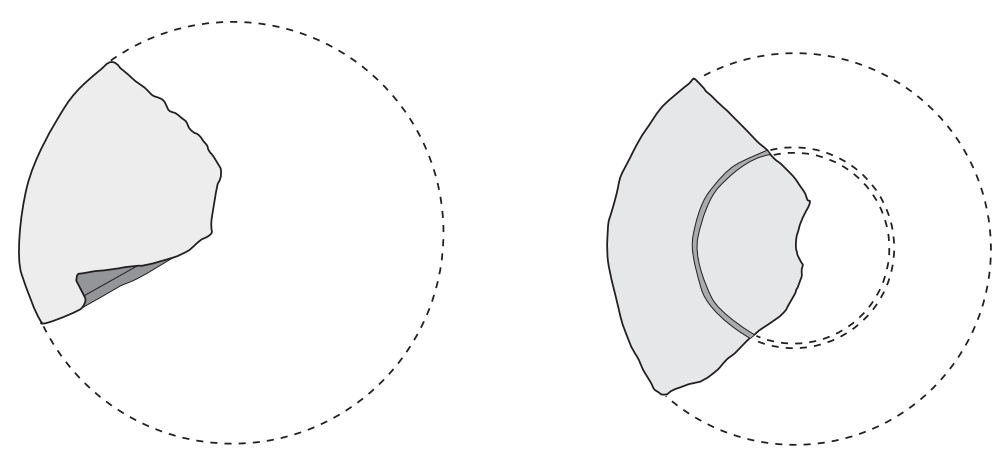

0

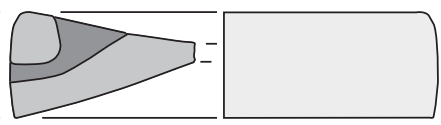

Reims (Marne) - rue Maucroix Ol 606, us 479

Calcaire à glauconie grossière Centre Inrap Reims

Florent Jodry, 2012

Étude ${ }^{\circ}{ }^{51-038}$
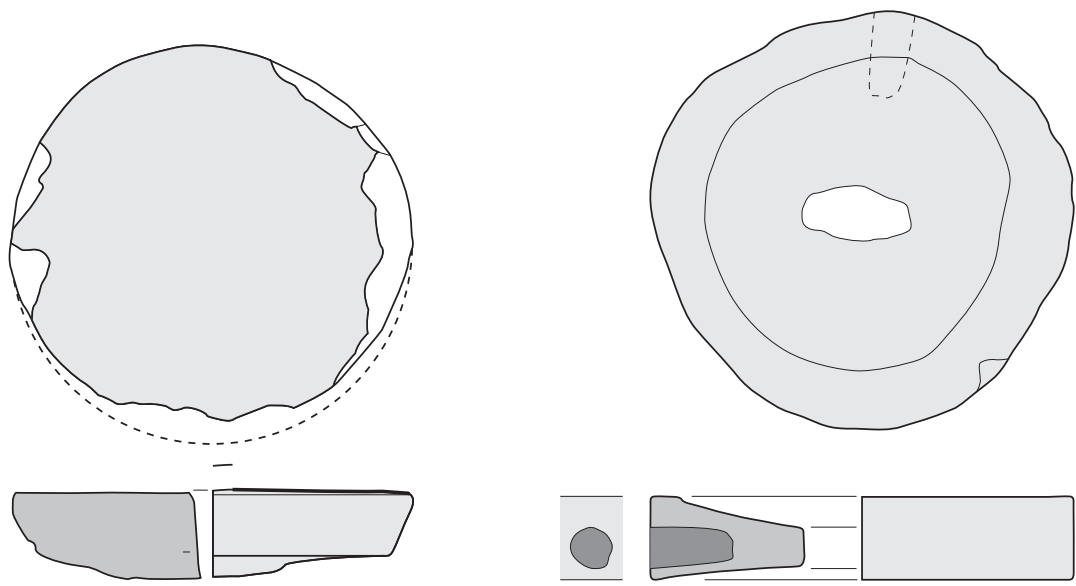

Reims (Marne) - rue Sarrail Calcaire à ditrupa

Dépôt SRA/Reims-Métropole

Paul Picavet Étude ${ }^{\circ}$ 51-304

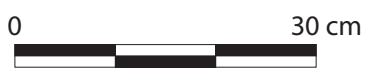

F11-21, sect3-tr.9

Calcaire à Cérithes

Dépôt Reims-Métropole

Florent Jodry, 2013

Étude $n^{\circ}$ 51-042

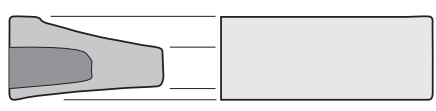

Reims (Marne)

Calcaire à Ditrupa

Reims-Musée Saint-Remi

Florent Jodry, 2014

Étude ${ }^{\circ}$ 51-313

Fig. 8 (suite). Sélection de meules manuelles et hydraulique de la ville de Reims. 
Sur les cinq types d'œils mis en évidence par B. Robin en 2011 (RoBIN, 2011), quatre sont répertoriés sur l'ensemble des catillus de Reims. Il s'agit des types 1, 2, 4 et 5 . Le type 3, associé à l'anille supérieure, n'est pas présent dans notre corpus.

Vingt catillus sont dotés d'un œil visible ou pouvant être renseigné par restitution et chaque type est représenté par des variétés qu'il convient de préciser:

Ces types de perforation sont fréquents, notamment dans la région (JODRY et alii, ce vol.) à l'exception du type $2 \mathrm{E}$ (circulaire flanqué de deux ouvertures rectangulaires) fortement représenté sur Reims et sur des calcaires gréseux et à Cérithes. Ce schéma avait déjà interpellé $\mathrm{Cl}$. Pommepuy dans son article sur le matériel de mouture de la vallée de l'Aisne (Pommepuy, 1999). Hormis le type 5B observé sur la meule hydraulique en basalte 51-016, toutes ces perforations sont visibles sur des meules manuelles en roche sédimentaire sans qu'il existe une correspondance entre la roche et le type d'œil.

Le type 5B est intéressant puisqu'il s'agit d'une meule hydraulique dotée de goulottes, tangentes à la perforation centrale, réalisées dans le but d'accueillir des tiges métalliques munies de crampons fichés dans la masse de la meule tournante (JODRY, 2011, p. 32). Ces crampons transmettent le mouvement rotatif à la meule et aident le centrage.

Afin d'actionner ces catillus différents types de manchon peuvent être réalisés. Nous en avons relevé trois sur les neuf de la typologie existante, le type 9 distinguant les catillus entiers qui ne présentent pas de trou de manchon (JODRY et alii, 2011). Il s'agit du manchon de type 1 (latéral horizontal partiel, très fréquent dans la vallée de l'Aisne), du type 4 (latéral oblique perforant) et du type 9 (absence). Sur les quarante-sept catillus de notre ensemble, seuls onze pièces possèdent encore un indice de perforation et ces derniers sont respectivement au nombre de cinq pour le type 1 , deux pour le type 4 et cinq pour le type 9 . Ces chiffres correspondent à ceux relevés sur les meules tournantes antiques de la région (JODRY et alii, ce vol.). La meule Re2 porte sur son flanc une trace d'oxydation qui peut être liée à un cerclage métallique pour le maintient d'un manche (JACCOTTEY et alii, 2008). Cette meule entre très certainement dans la catégorie du type 9.

La même remarque formulée pour les œils peut être faite pour les manchons: aucune correspondance n'a pu être relevée entre la roche et le type de manchon et entre le type de manchon et d'œil.

La surface active des meules de notre ensemble accueille dans quatre cas un habillage raisonné : habillage simple (simple droit et simple courbe sur arkose de type Haybes/Macquenoise et calcaire gréseux) pour deux pièces et un habillage composé pour les deux autres : composé droit et composé courbe uniquement sur grès de Fosses-Belleu comme à Meaux (LEPAREUX-COUTURIER, 2011, p. 423).
La meule 51-028 porte un rhabillage double puisqu'elle présente successivement des segments rectilignes d'un habillage composé droit et des lignes courbes d'un habillage composé courbe.

Pour terminer nous devons mentionner la présence de sept meules, découvertes sur différents sites, remployées probablement en polissoir. Ces sept pièces, réalisées dans différentes roches sédimentaires portent sur leur surface active (pour les meta) ou sur leur surface supérieure (pour les catillus) des cupules ovoïdes ou plus géométriques, profondes de quelques centimètres. La fonction de polissage est avancée en raison de l'absence d'impacts mais sans autres indices précis.

\section{CONCLUSION}

L'occupation augustéenne trace les contours de la capitale de la Gaule Belgique Durocortorum et la dote d'une superficie enceinte de près de 600 ha. Les nombreuses fouilles menées au cœur de cette cité ont permis de prélever plus de 70 meules à entraînement manuel ou mécanique.

Ce chiffre reste toutefois modeste pour une superficie fouillée de près de $50000 \mathrm{~m}^{2}$ (en comptabilisant l'ensemble des opérations) et l'on ne peut que constater une disparité entre le nombre de meules découvertes et les surfaces étudiées. En effet, il est étonnant d'observer que certains îlots urbains ne livrent aucune trace d'éléments de mouture, par ailleurs très nombreux dans certains secteurs comme sur les fouilles du boulevard Henrot ou de la rue Maucroix.

À l'heure actuelle aucune pièce n'est associée avec certitude à un lieu de traitement des céréales. Malgré ces nombreux contextes de rejet dans lesquels les meules ont été mises au jour, ces dernières restent toutefois les témoins d'une activité qui se déroulait très probablement à proximité. Il en ressort toutefois que cette activité, peu centralisée sur les monuments publics du cœur de la cité, est présente sur la frange occidentale de la ville le long d'axes où les quartiers artisanaux fleurissent.

Malgré la découverte de nombreuses meules de moulins manuels, $87 \%$ du corpus, nous ne sommes pas assurés que cette activité reste concentrée dans l'espace domestique. En effet, la découverte de quarante-deux meules, $70 \%$ du corpus manuel, sur les sites du boulevard Henrot ou des rues Maucroix et Rockefeller, nous incite à penser que cette activité peut être pratiquée par de nombreux artisans meuniers.

À ce titre nous pouvons proposer deux schémas interpénétrables suivant peu ou prou l'évolution de la ville.

Le premier schéma suit une structure simple d'évolution des techniques artisanales de traitement des céréales où les meules domestiques manuelles, destinées à une production domestique, sont progressivement remplacées par des meules de grand diamètre, principaux témoins d'une production de masse «à la romaine»(PICAVET, 2011). 
Cependant ce schéma trop simpliste et caricatural peut être contesté par un second modèle dans lequel des éléments domestiques et artisanaux coexistent.

Ce schéma, débutant au Haut-Empire, intègre à la fois des moyens de production destinés à subvenir aux besoins quotidiens de familles comme le suggère les découvertes issues des fouilles du Tramway-secteur 5 ou affectés à la production artisanale (boulevard Henrot, rue Maucroix). Nous suggérons par conséquent que la multiplication des moulins concentrés dans certaines parties de la cité sert probablement à nourrir une population ne disposant pas de moyens financiers pour l'achat de moulins domestiques.

En revanche le Bas-Empire semble ouvrir la voie à un nouveau modèle de consommation où les meules de grands diamètres, associées à des structures de production plus importantes, sont repoussées progressivement vers l'extérieur de la ville en zone péri-urbaine (bd Joffre, rue SaintSymphorien, rue de Cernay). La production semble passer alors entre les mains d'artisans spécialisés produisant de grandes quantités de farine.

Ce modèle de production témoin de la romanisation se vérifie également à travers le choix des roches dans lesquelles sont réalisées les meules. La présence durant le Haut-Empire de calcaires locaux laisse la place, au BasEmpire à un système d'importation de roches, sans doute plus efficaces, provenant des carrières extra-régionales des Ardennes ou de l'Eifel. 


\section{BON DE COMMANDE}

LES MeUles du NÉOlithiQUe À L'ÉPOQUe MÉdiÉVALE : TECHNIQUE, CULTURE, DIFFUSION

Actes du $2^{\text {ème }}$ colloque du Groupe Meule,

Reims, du 15 au 17 mai 2014

Sous la dir. d'O. Buchsenschutz,

S. Lepareux-Couturier et G. Fronteau

Un volume de 528 pages, nombreuses illustrations n. \& b. et couleur, format $21 \times 29,7 \mathrm{~cm}$

\section{Prix : 35 Euros}

Nom : .

Prénom :

Adresse :

Code postal. ...Ville

\section{Adresse de facturation, si différente :}

Adresse :

Code postal ...Ville

désire recevoir :

..... exemplaire(s) à $35 €$, soit

+ frais de port $7 €$ pour 1 vol.

Date et signature

Réglement à l'ordre de :

\section{SOCIÉTÉ ARCHÉOLOGIQUE DE L'EST}

CCP 1268-33 A, DIJON

RIP : Ét. 20041 - G. 01004 - Nº 0126833 A025 - clé : 85

IBAN : FR 5920041010040126833 A025 85

BIC/SWIFT : PSSTFRPPDIJ

Pour létranger : paiement par chèques, mandat ou

virement international uniquement.

\section{RÉSUMÉ}

Ce colloque s'inscrit dans la dynamique du PCR "Évolution typologique et technique des meules du Néolithique à l'an mille sur le territoire français ». Il fait suite à la table ronde de Saint-Julien-sur-Garonne publiée en 2011, qui avait présenté les normes descriptives, validé les premiers résultats obtenus sur le fonctionnement des meules et défini les pistes d'une analyse typologique. Ce volume présente le dépouillement systématique des données de Champagne-Ardenne effectué avec l'aide des archéologues et des conservateurs de cette région. C'est la première fois qu'une action de ce type est effectuée. Elle révèle la potentialité d'un espace donné en matière de meules et mesure l'important travail nécessaire pour documenter ce corpus. Cette étude locale est accompagnée de contributions sur d'autres régions et pays, particulièrement de la Méditerranée. Concernant l'analyse des bases de données nationales, le colloque de Reims a été l'occasion de mettre en relation les différentes variables, de confirmer l'efficacité des ou tils mis en place, de présenter des méthodes d'an alyses statistiques et cartographiques globales d'ores et déjà opérantes et de corriger rapidement les éventuels dysfonctionnements. L'intérêt de l'association systématique des analyses typologiques et géologiques des meules a été confirmé. Plus-value essentielle à notre recherche collective, un certain nombre de questions techniques et chronologiques ont été résolues par le biais d'analyses croisées fondées sur des contextes archéologiques fiables et une analyse rigoureuse et homogène des géomatériaux. Le succès de cette rencontre doit beaucoup à l'accueil du laboratoire du GEGENAA (EA3795) au sein de l'université de Reims, particulièrement efficace et chaleureux.
Cet ouvrage a été édité dans le cadre de l'UMR 6298 ArTeHiS, à l'Université de Bourgogne avec l'aide du Ministère de la Culture et de la Communication (D.R.A.C. de Champagne-Ardenne), de l'École Normale Supérieure de Paris, de l'Université de Reims Champagne-Ardenne (EA 3795 GEGENAA) et de l'Institut national de Recherches archéologiques préventives

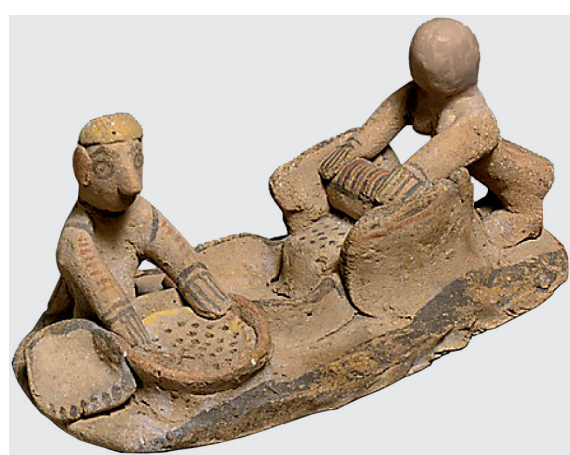

- - ००००० - -

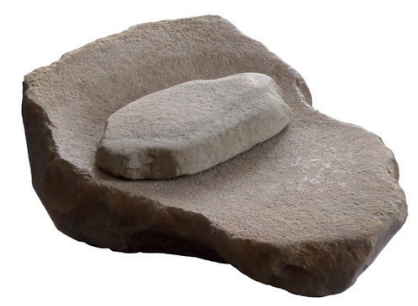

Bon de commande à retourner à :

REVUE ARCHÉOLOGIQUE DE L'EST

Université de Bourgogne, UMR 6298 ARTeHIS

6, boulevard Gabriel, F 21000 DIJON

Tél. 03.80.39.55.66

EMail : claire.touzel@u-bourgogne.fr

Seules les commandes accompagnées d'un réglement seront honorées; pour les administrations, d'un bon de commande.

Attention: les suppléments à la R.A.E. ne se reçoivent pas par abonnement, mais font l'objet d'une commande spécifique.

Tous les suppléments sur : http://rae.revues.org/ 


\section{SOMMAIRE}

Pr. Vincent BARBIN

Introduction

Alain KOEHLER

Introduction

Pr. Jean-Pierre BRUN

Des meules et des hommes.

\section{(S) \\ Première partie \\ LES MEULES À GRAIN DU NÉOLITHIQUE À L'ÉPOQUE MÉDIÉVALE EN CHAMPAGNE-ARDENNE}

O. BuCHSENSCHUTZ, C. HAMON et L. JACCOTTEY

Étude des meules en Champagne-Ardenne.

G. Fronteau, F. Boyer, M. Frouin., L. Jaccottey, S. LepareuX-Couturier, A. Milleville et P. Picavet

Les principales pierres meulières utilisées en Champagne-Ardenne : gisements, matériaux, utilisations.

C. Hamon, V. Brisotto, G. Fronteau, L. Jaccottey et C. Monchablon, avec la coll. de K. Cherfa,

S. Cousseran-Néré, K. Donnart, M. Frouin, J.-L. Gisclon, S. LePareuX-Couturier, P. PicAVet et B. Robin Les moulins va-et-vient du Néolithique à l'âge du Bronze en Champagne-Ardenne.

L. Jaccottey, G. Fronteau, O. Buchsenschutz, F. Jodry et M. SAurel, avec la coll. de S. Cousseran-NéRé, S. Galland, C. Garcia, J.-L. Gisclon, S. LepareuX-Couturier, C. Monchablon, B. Robert et B. Robin Les meules de l'âge du Fer en Champagne-Ardenne

F. Jodry, L. Jaccottey, G. Fronteau, S. LePareuX-Couturier, P. PiCAVET et B. Robin

Les meules antiques de Champagne-Ardenne

N. MinVIELle-LAROUSSE, L. JACCOTTEY et S. LEPAREUX-COUTURIER

Les outils de mouture en Champagne-Ardenne à l'époque médiévale.

M. ETCHART-SALAS

Le matériel de mouture de Bezannes (Marne) du Néolithique à la période gallo-romaine: synthèse d'une quinzaine d'années d'archéologie préventive.

O. Buchsenschutz, G. Fronteau, L. Jaccottey, F. Jodry et M. SAURel

Les meules gauloises d'Acy-Romance (Ardennes), La Warde.

F. Jodry, P. Rollet, P. PicAVet, Fr. Moiret et G. FronteAu, avec la coll. d'A. BALMELle

Meules antiques de Reims 


\section{Deuxième partie \\ CARRIÈRES, MATÉRIAUX, PRODUCTIONS}

T. J. ANDERSON et L. JACCOTTEY

Vers une classification et une normalisation des termes pour définir les carrières de meules.

G. Fronteau, F. Boyer, O. Buchsenschutz, S. LepareuX-Couturier, C. HAMON et L. JACCOTtey

Caractérisations et indexations des variétés des pierres meulières: de l'étude pétrographique à la définition de types simplifiés au sein d'une base de données géoréférencées. . .

L. JACCOTTEY, F. BOYER et S. ALIX

L'atelier antique de façonnage de meules rotatives d'Autun (Sâ̂ne-et-Loire).

F. JODRY et J.-M. HOLDERBACH

La carrière d'extraction de meules en grès vosgien du Purpurkopf à Rosheim (Bas-Rhin)

L. JACCOTTEY et A. MiLLEVILLE

7000 ans d'exploitation meulière dans le massif de La Serre.

S. Lepareux-Couturier, F. Boyer, G. Fronteau, C. Garcia, C. Hamon, C. Monchablon,

P. PICAVET et B. ROBIN

Les productions de meules en grès de Fosses-Belleu dans le Bassin parisien : typologie, chronologie, diffusion.... 213

S. LepareuX-Couturier, G. Fronteau, C. Monchablon et B. Robin

Les productions de meules en meulière dans le Bassin parisien : typologie, chronologie, diffusion

L. JACCOTTEY, F. JODRY et T. ANDERSON

Les productions de meules en "grès des Vosges» de La Tène finale à la fin de l'Antiquité: typologie, chronologie,

diffusion. ...

P. PicAVet, G. Fronteau, V. Le QuelLeC et F. Boyer

Les productions de meules en grès dévonien dit «arkose» d'Haybes/Macquenoise de la fin de l'âge du Fer à l'Antiquité tardive dans le nord de la Gaule : caractérisation typologique, chronologie et diffusion. ...

\section{क \\ Troisième partie \\ TYPOLOGIE CROISÉE, ANALYSES STATISTIQUE ET CARTOGRAPHIQUE}

C. Hamon, V. Brisotto, A. Milleville, C. Monchablon, L. Aubry, S. Cousseran-Néré, L. Jaccottey,

K. DONNART, G. FronteaU et J.-L. Gisclon

Évolution des meules va-et-vient du Néolithique à l'âge du Fer en France: exploitation de la base de données du PCR Meule

C. HAMon, V. Brisotto, L. Jaccottey, C. Monchablon, S. Cousseran-NéRÉ, K. Donnart, T. Duda,

J.-L. GiSCLON et A. MILLEVILLE

Les molettes débordantes du Néolithique : définition et premier état des lieux

S. LEPAREUX-COUTURIER et B. Robin, avec la coll. d'O. BUCHSENSChUTZ et G. FrontEAU

Évolution des meules rotatives de l'âge du Fer au Moyen Âge en France: exploitation de la base de données du PCR Meule

S. LePAREUX-COUtURIER, E. HARTOCH et P. PiCAVET, avec la coll. de F. JODRY

Le rayonnage des meules rotatives antiques dans le nord-est de la Gaule. 


\section{Quatrième partie \\ ÉTUDES DE CAS EN FRANCE}

T. DUDA

Étude du mobilier de mouture et du macro-outillage du camp néolithique de Chassey, 'La Redoute',

Saône-et-Loire ............................................................. 351

S. COUSSERAN-NÉRÉ et J.-L. GISClON, avec la coll. de L. JACCOTTEY

Les outils de mouture du site chasséen de Montélimar 'Daurelle-Autajon' (Drôme)

J.-L. GISCLON, avec la coll. de S. COUSSERAN-NÉRÉ, L. JACCOTTEY et P. HÉNON

L'outillage de mouture va-et-vient des Feuilly à Saint-Priest (Rhône)

V. GEORGES, L. JACCOTTEY et B. ROBIN

Les molettes à rainure: nouveaux indices sur le perfectionnement du matériel de mouture dans le bassin

de la Loire à l'âge du Fer..

V. GEORGES et L. JACCOTTEY, avec la coll. de J.-C. BÉFORT

Un fragment de meule rotative en contexte précoce à Lijay dans les Monts du Forez

(fin IVe-début III ${ }^{e}$ s. av. J.-C.)

P. PICAVET, avec la coll. de G. FronteAu et C. FonT

Distribution des matériaux meuliers sur un transect nord-sud à travers la France septentrionale:

les meules rotatives gauloises, gallo-romaines et alto-médiévales du tracé du canal Seine - Nord Europe

G. Asselin, G. Brkojewitsch, S. Marquié, J. Maujean et L. Pruneyrolles

Outils de mouture et de broyage d'époque tibérienne: la fouille préventive de la Rue Paille-Maille à Metz.

(Moselle)

N. MiNVIELLE LAROUSSE

Les meules du moulin à minerai médiéval de Brandes-en-Oisans

\section{Cinquième partie}

\section{ÉTUDES DE CAS EN EUROPE ET DANS LE MONDE}

S. Reniere, W. De ClercQ, R. Dreesen, V. CNudde, T. De Kock et E. Goemaere

Recherches sur des meules romaines dans un paysage dépourvu de ressources lithiques:

premier bilan d'une analyse multidisciplinaire dans la Civitas Menapiorum (Belgique)

C. Chaigneau

Le moulin «délien» : apport du corpus de Mégara Hyblaea (Sicile). . .

A. CARBILlet et F. JODRY

Les outils de mouture du Palais d'Amathonte (Chypre) à l'âge du Fer : premiers résultats.

N. AlONSO et R. FRANKEL

A survey of ancient grain milling systems in the Mediterranean

LI GUOQIANG

La meule, la cueillette et la proto-domestication des millets depuis le Paléolithique supérieur jusqu'au début du Néolithique dans la Chine septentrionale 
O. BuchsenschutZ, G. Fronteau et S. LePAREuX-COUTURIER

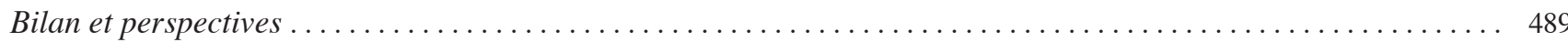

Bibliographie générale ....................................................... 493

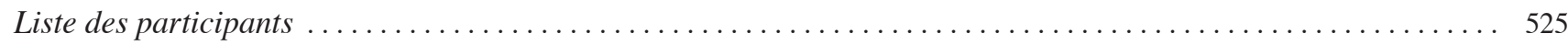

